\title{
Methods for Wild Pig Identifications from Moving Pictures and Discrimination of Female Wild Pigs based on Feature Matching Methods
}

\author{
Kohei Arai 1 \\ Graduate School of Science and Engineering \\ Saga University \\ Saga City, Japan \\ Indra Nugraha Abdullah 2 \\ 2 Jakarta Office, Yamaha Co. Ltd. \\ Jakarta, Indonesia
}

\author{
Kensuke Kubo 3 \\ 3 Fujitsu Kyushu Network Technologies, Ltd. \\ Fukuoka Japan \\ Katsumi Sugawa 3 \\ 3 Fujitsu Kyushu Network Technologies, Ltd. \\ Fukuoka Japan
}

\begin{abstract}
Methods for wild pig identifications and discrimination of female wild pigs based on feature matching methods with acquired Near Infrared: NIR moving pictures are proposed. Trials and errors are repeated for identifying wild pigs and for discrimination of female wild pigs through experiments. As a conclusion, feature matching methods with the target nipple features show a better performance. Feature matching method of FLANN shows the best performance in terms of feature extraction and tracking capabilities.
\end{abstract}

Keywords-OpenCV; Canny filter; Template matching; Feature matching

\section{INTRODUCTION}

Wildlife damage in Japan is around 23 Billion Japanese Yen a year in accordance with the report from the Ministry of Agriculture, Japan. In particular, wildlife damages by deer and wild pigs are dominant (10 times much greater than the others) in comparison to the damage due to monkeys, bulbuls (birds), rats. Therefore, there are strong demands to mitigate the wildlife damage as much as we could. It, however, is not so easy to find and capture the wildlife due to lack of information about behavior. For instance, their routes, lurk locations are unknown and not easy to find. Therefore, it is difficult to determine the appropriate location of launch a trap.

The purpose of this research work is to identify the wildlife, in particular, wild pigs for mitigation of wildlife damage. In particular, it is effective to capture female wild pigs (wild boar lays the child) for mitigation of wildlife damage. Therefore, there are very strong demands of capturing female wild pigs.

In order to identify the wild pigs and discriminate female wild pigs from the moving pictures acquired with Near Infrared: NIR camera, computer vision of technologies are utilized. First, target of wild pigs is attempted to extract from the moving pictures. Contour extraction and edge extraction are attempted. Secondly, background and target are attempted to separate. Using a template of nipple image (a small portion of image), discrimination of female wild pigs is attempted.
Then feature matching methods are used for female wild pig discriminations with nipple features acquired from the moving pictures.

The following section describes research background followed by the proposed methods for wild pig identification and discrimination of female wild pigs. Then experiments are described followed by conclusion with some discussions.

\section{RESEARCH BACKGROUND}

According to the West, B. C., A. L. Cooper, and J. B. Armstrong. 2009. Managing wild pigs: A technical guide. Human-Wildlife Interactions Monograph 1:1-55 ${ }^{1}$, there are the following wild pig damages,

\section{Ecological}

Impacts to ecosystems can take the form of decreased water quality, increased propagation of exotic plant species, increased soil erosion, modification of nutrient cycles, and damage to native plant species [1]-[5].

Agricultural Crops

Wild pigs can damage timber, pastures, and, especially, agricultural crops [6]-[9].

\section{Forest Restoration}

Seedlings of both hardwoods and pines, especially longleaf pines, are very susceptible to pig damage through direct consumption, rooting, and trampling [10]-[12].

Disease Threats to Humans and Livestock

Wild pigs carry numerous parasites and diseases that potentially threaten the health of humans, livestock, and wildlife [13]-[15].

Humans can be infected by several of these, including diseases such as brucellosis, leptospirosis, salmonellosis, toxoplasmosis, sarcoptic mange, and trichinosis. Diseases of

\footnotetext{
1 www.berrymaninstitute.org/publications,
} 
significance to livestock and other animals include pseudorabies, swine brucellosis, tuberculosis, vesicular stomatis, and classical swine fever [14], [16]-[18].

There also are some lethal techniques for damage managements. One of these is trapping. It is reported that an intense trapping program can reduce populations by 80 to $90 \%$ [19]. Some individuals, however, are resistant to trapping; thus, trapping alone is unlikely to be successful in entirely eradicating populations. In general, cage traps, including both large corral traps and portable drop-gate traps, are most popular and effective, but success varies seasonally with the availability of natural food sources [20]. Cage or pen traps are based on a holding container with some type of a gate or door [21].

\section{PROPOSED METHOD AND SYSTEM}

\section{A. Proposed System}

Fig.1 shows an example of the system for trapping and capturing of wild pigs which consists of the trap cage and the video camera.

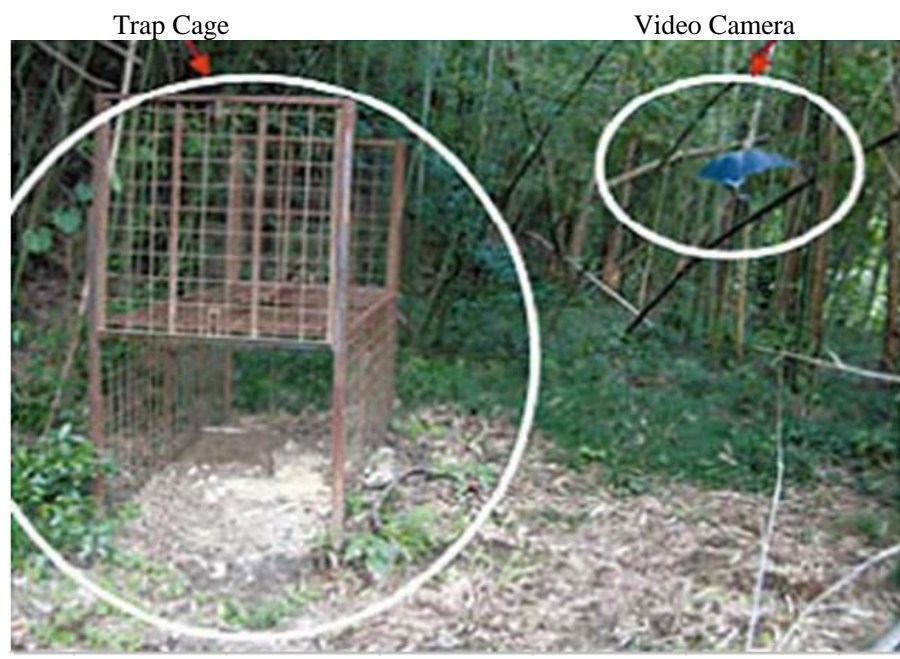

Fig. 1. Proposed system for trapping and acquiring moving picture of wild pigs

In the trap cage, there is bait. When wild pigs get in the trap cage, ultrasonic sensor sensed them. Then the entrance doors are shut downed. These processes are monitored and captured with the near infrared video camera with near infrared Light Emission Diode: LED. Because wild pigs are active in nighttime, Near Infrared: NIR camera with NIR LED is used.

The proposed system for trapping of wild pigs and for capturing their moving pictures is illustrated in Fig. 2.

There are two ultrasonic sensors which are attached at the front and the back ends of the cage. When wild pigs get in the cage, then they are sensed with the ultrasonic sensors. Meantime, trap obstruction is activated. Drop-gates are then shut downed immediately after they are sensed with the ultrasonic sensors. Thus the wild pigs are trapped in the cage. These processes are monitored and captured with NIR camera with NIR LED. The captured moving pictures are transmitted through Bluetooth and then the transmitted moving pictures are transferred to the data collection center through $\mathrm{WiFi}$ networks or LAN. There are sensor control and battery box as well as solar panel for electricity supply.

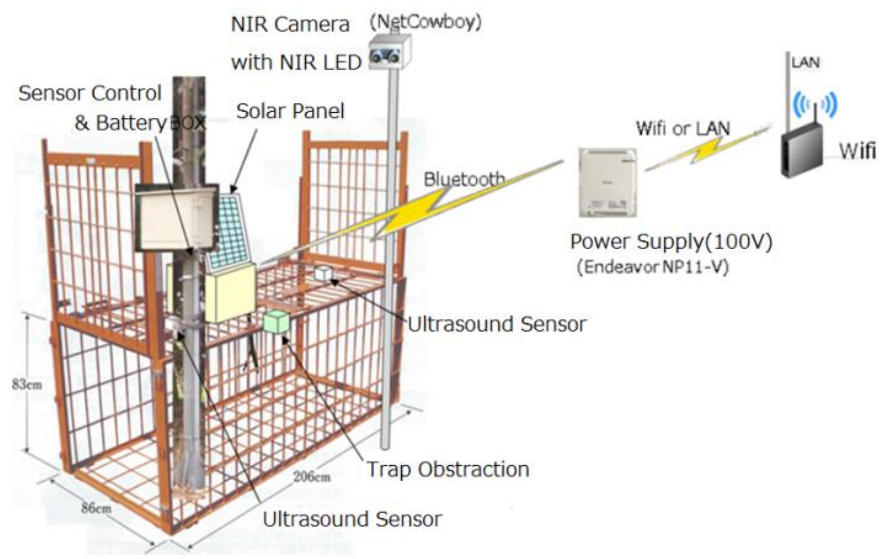

Fig. 2. Proposed system for trapping of wild pigs and for capturing their moving pictures

Outlooks of the NIR camera (NetCowboy) with NIR LED and ultrasonic sensors are shown in Fig. 3 (a) and (b), respectively. Meanwhile, specifications of these camera and sensor are shown in Table 1 and 2, respectively

TABLE I. SPECIFICATION OF NIR CAMERA (NETCOWBOY)

\begin{tabular}{|c|c|}
\hline Pixel & $1.3 \mathrm{M}$ \\
\hline Resolution & $1280 \times 1024$ \\
\hline Frame rate & $1280 \times 1024: 7.5 \mathrm{fps}, 640 \times 480: 30 \mathrm{fps}$ \\
\hline Dimension & $52 \mathrm{~mm}(\mathrm{~W}) \times 65 \mathrm{~mm}(\mathrm{D}) \times 70 \mathrm{~mm}(\mathrm{H})$ \\
\hline Weight & $85 \mathrm{~g}$ \\
\hline Operating condition & $0-40 \mathrm{deg} . \mathrm{C}$ \\
\hline Interface & USB 2.0 \\
\hline IR Illumination & 7 NIR LED \\
\hline
\end{tabular}

TABLE II. FEATURES OF Ultrasonic RANGE FindeR ${ }^{2}$

\begin{tabular}{|l|l|}
\hline Supply Voltage & $5 \mathrm{~V}$ (DC) \\
\hline Supply Current & $30 \mathrm{~mA}($ Typ), $35 \mathrm{~mA}$ (Max) \\
\hline Range & $3 \mathrm{~cm}$ to $3 \mathrm{~m}$ \\
\hline Input Trigger & Positive TTL pulse, $2 \mu \mathrm{S}$ min, $5 \mu \mathrm{S}$ (Typ) \\
\hline Echo Pulse & Positive TTL pulse, $115 \mu \mathrm{S}$ to $18.5 \mathrm{mS}$ \\
\hline Echo Hold-off & $750 \mu \mathrm{S}$ from fall of Trigger pulse \\
\hline Burst Frequency & $40 \mathrm{kHz}$ for $200 \mu \mathrm{S}$ \\
\hline Delay before next measurement & $200 \mu \mathrm{S}$ \\
\hline Dimension & $22 \mathrm{~mm} \mathrm{H}$ x $46 \mathrm{~mm} \mathrm{~W} \mathrm{x} 16 \mathrm{~mm} \mathrm{D}$ \\
\hline
\end{tabular}

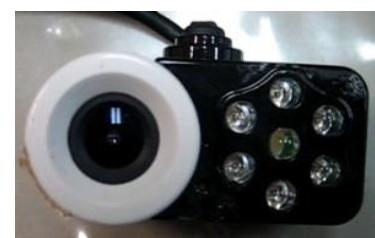

(a)NIR camera

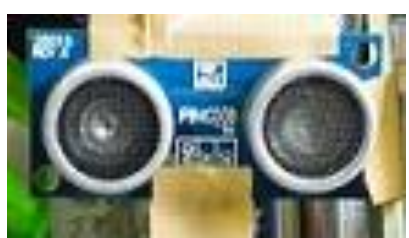

(b)Ultrasonic sensor
Fig. 3. Outlook of NIR camera with NIR LED and ultrasonic sensor used in the proposed system for trapping and capturing of wild pigs

\footnotetext{
2 Parallax ultrasonic sensor,

http://www.parallax.com/dl/docs/prod/acc/PingDocs.pdf
} 


\section{B. Proposed Moving Picture Analysis Methods}

Moving pictures are acquired with high resolution mode of 1280 by 1024 pixels. Therefore, frame rate is $7.5 \mathrm{fps}$. OpenCV is used for acquisition, processing, and analysis because it is totally easy to use. OpenCV is an open source computer vision library which is written in $\mathrm{C}$ and $\mathrm{C}++$ and runs under Linux, Windows, and Mac OS X. It can be downloaded from http://sourceforge.net/projects/opencvlibrary

There so many library software for image processing and analysis. First, object has to be extracted from the moving picture. Then object contour has to be extracted. For the contour extraction and tracing, Canny filter related spatial filters are attempted. After that, it would be better to remove the background. The following background removals is attempted,

\section{cv2.createBackgroundSubtractorMOG()}

In order to discriminate female wild pigs, template matching method is applied with a template of small portion of nipple images. The following correlation functions are attempted for template matching,

\section{CV_TM_SQDIFF , CV_TM_SQDIFF_NORMED , CV_TM_CCORR , CV_TM_CCORR_NORMED , CV_TM_CCOEFF, CV_TM_CCOEFF_NORMED}

Also feature matching methods are applied for discrimination of female wild pigs. There are many feature matching methods in the OpenCV library. A couple of feature matching methods are attempted for the discriminations. The followings are typical feature matching methods which are provided from OpenCV,

- BruteForce

- BruteForce-L1

- BruteForce-SL2

- BruteForce-Hamming

- BruteForce-Hamming(2)

- FlannBased

The FlannBasedMatcher interface is used in the proposed method in order to perform a quick and efficient matching by using the FLANN (Fast Approximate Nearest Neighbor Search Library). Also Brute-Force matcher which is simple matching method is used in the proposed method. It takes the descriptor of one feature in first set and is matched with all other features in second set using some distance calculation. For both, feature descriptor is needed. Speeded-up Robust Feature: SURF is used in the proposed method.

\section{EXPERIMENTS}

\section{A. Preliminary Image Processing}

One shot image of the acquired moving pictures is shown in Fig.4 as an example. This is a female wild pig on the route from habitat area to go to the calms feed. Wild boar children are followed by the female wild pig. By using the difference between the current and the previous frame of wild pig (targeted object), it is possible to extract the female wild pig. Also, it is possible to remove the background by frame by frame. Fig.5 shows the resultant image of the background removals.

Edge and contour extractions are attempted with Canny and sharp Canny filters. Fig.6 (a) shows the resultant image of Canny filter while Fig.6 (b) shows that of the sharp Canny filter. In the process, lower and higher thresholds are adequately set obviously. Through a comparison between Fig.6 (a) and (b), sharp Canny filter seems superior to Canny filter. It, however, is not sufficient for extraction.

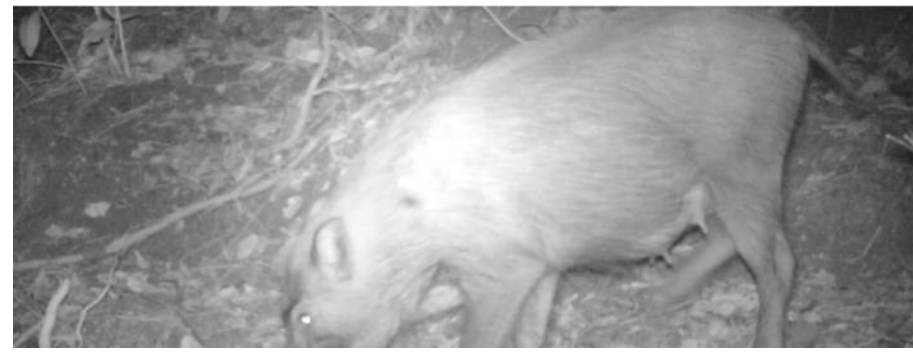

Fig. 4. Portion of original image of the targeted object of female wild pig in concern

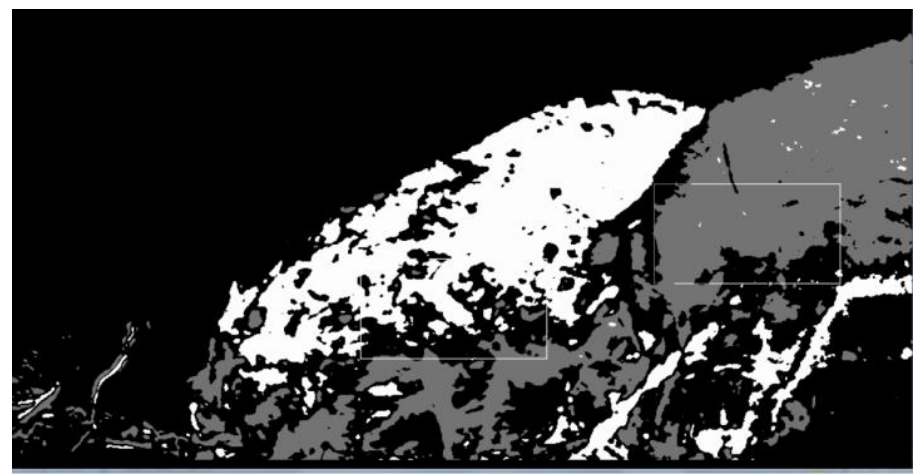

Fig. 5. Resultant image of background removal from the original image in frame by frame basis

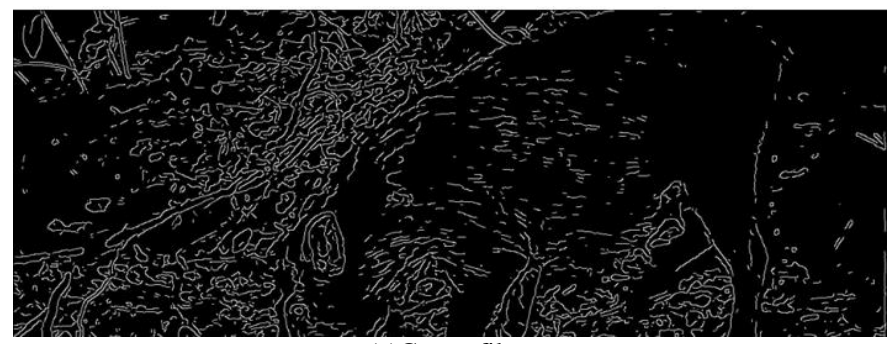

(a)Canny filter

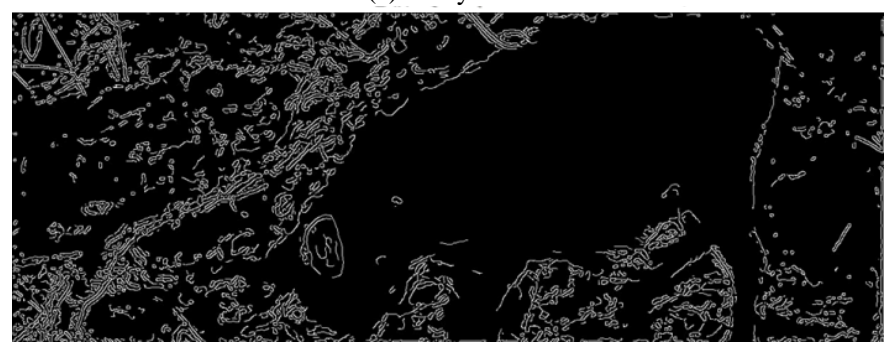

(b)Sharp Canny filter

Fig. 6. resultant images of edge and contour extractions 


\section{B. Descrimination of Female Wild pigs}

Secondly, discrimination of female wild pigs is attempted with template matching and feature matching. Fig.7 shows an example of template image of nipple which indicates female wild pigs.

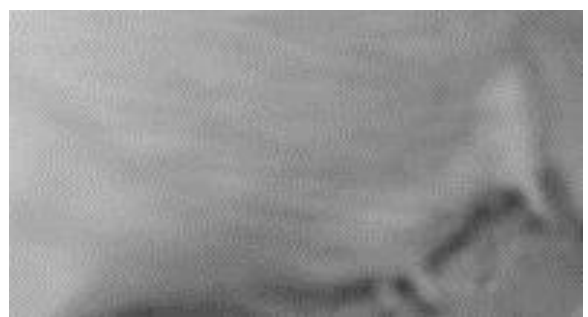

Fig. 7. Template image of nipple which is an indicator of female wild pigs

By using template matching software which is provided by OpenCV, nipple feature is matched and tracked. An example of template matching image with template image is shown in Fig.8. It seems does work well for female wild pig discrimination and tracking. It, however, does not work so well when the wild pig moves so fast and the portion of nipple is occluded and disappeared which are shown in Fig.9 (a) and (b), respectively. Also influence due to the both target moving speed and occlusion of the different object behind the other object is shown in Fig.9 (c).

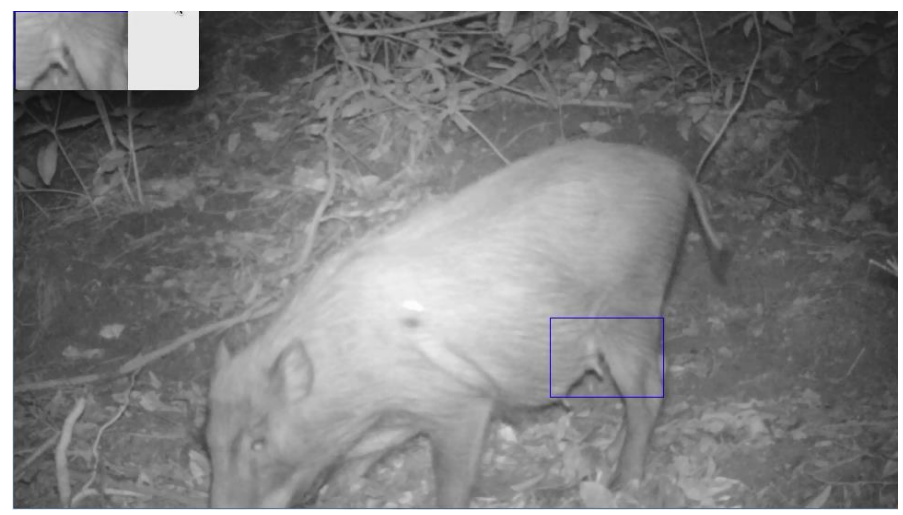

Fig. 8. Example of resultant image of template matching with the template image of nipple portion of image

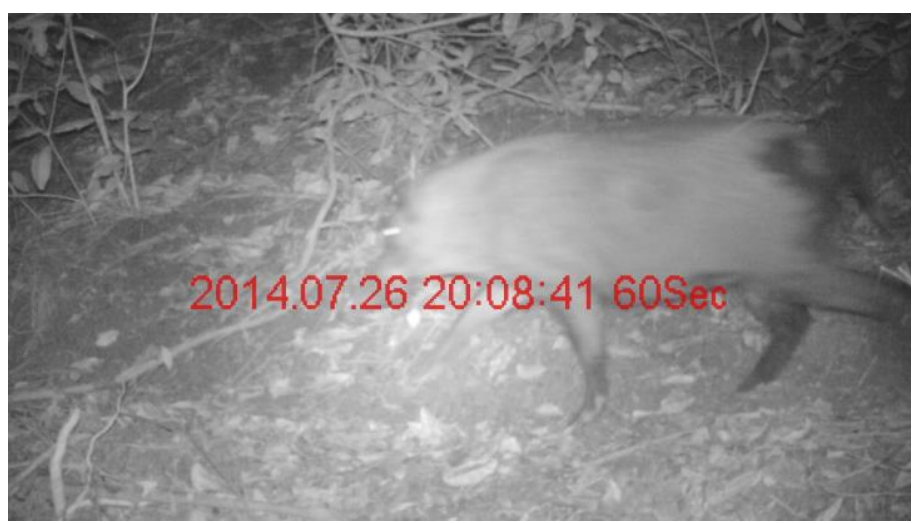

(a)Move so fast

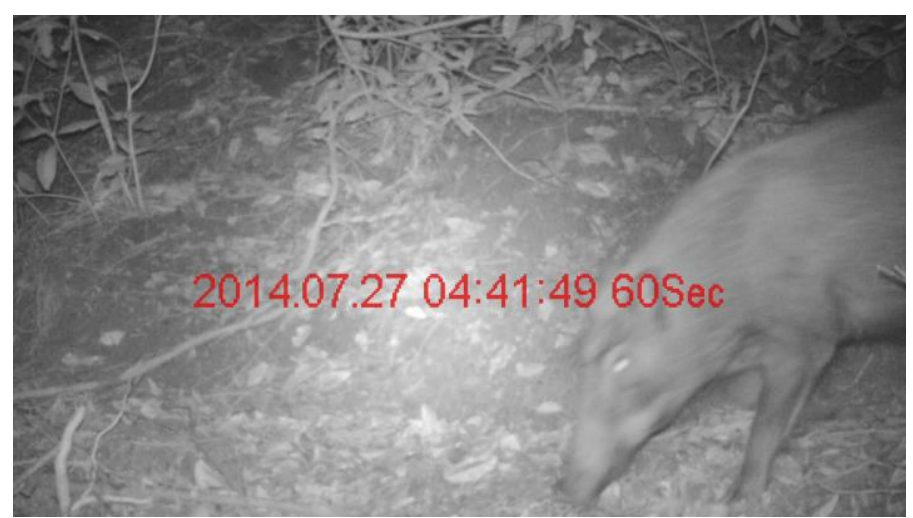

(b)Nipple portion cannot be seen

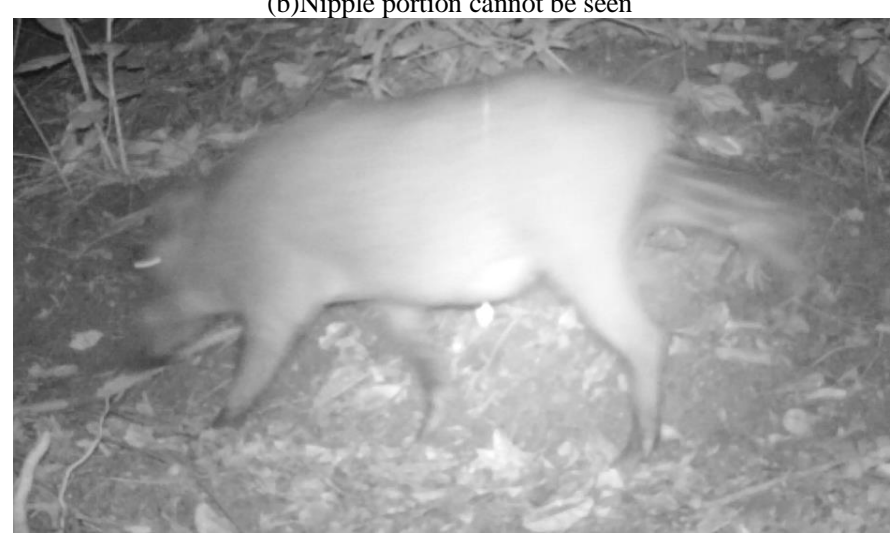

(c)Cub behind male adult wild pig

Fig. 9. Influences due to target speed and occlusion in template matching

Other than these, feature matching methods are attempted for discrimination of female wild pigs. In order to describe the feature of female wild pigs, Scale-Invariant Feature Transform: SIFT and SURF based feature descriptors are used for representation of nipple features. SURF descriptor based feature matching and tracking is attempted. Fig.10 shows an example of the resultant image of SURF feature matching.

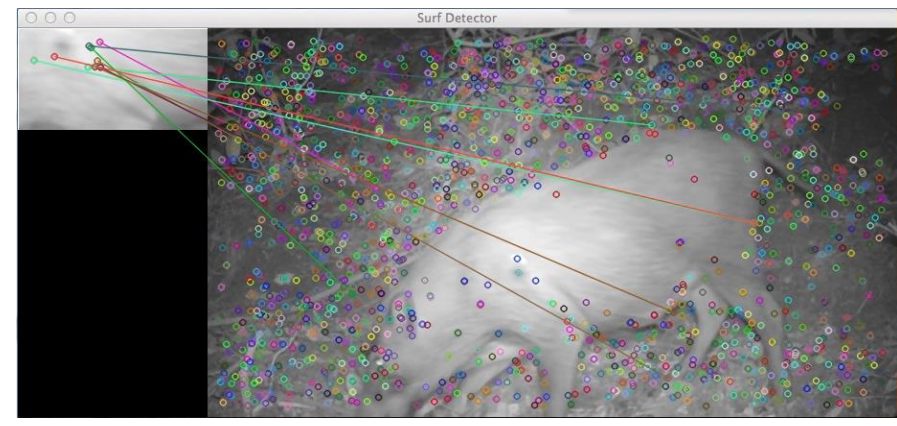

Fig. 10. Resultant image of SURF based feature description and feature matching

The SURF based feature description and feature matching is not good enough for feature tracking. Sometime it works well, but it does not work well as shown in Fig.10. Therefore, another feature matching methods are attempted after that. 


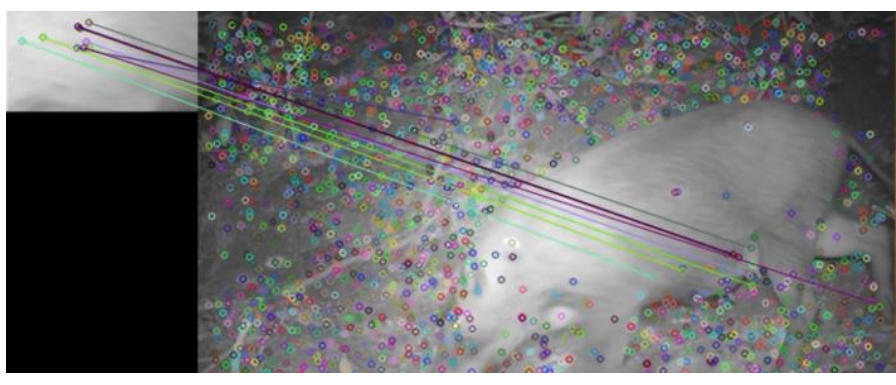

Fig. 11. Example of the resultant image of FLANN

Those are SURF Brute-Force and FLANN. The performance of discrimination is almost similar between both. Fig. 11 shows an example of the resultant image of FLANN.

Nipple of features is matched so well. In particular, tracking capability of the FLANN feature matching is superior to the other template matching and SURF matching as well as SURF Brute-Force matching.

\section{Proposed System for Wild Pig Montoring Hardware System}

One of the issues for damage management due to wild pigs is how to count the number of female wild pigs in the area in concern. Although the proposed methods and systems above work well, the hardware system is costly. The hardware system proposed here is cheap version of the system for monitoring the number of female wild pigs. Because the areas where suffers from wild pig damage are situated almost all over the Japanese island. Such situation is common to the countries in the world. Therefore, the cheap version of hardware system for monitoring is required.

Android tablet terminal which equipped communication capability (Bluetooth, WiFi) and camera is not so expensive. For instance, Android tablet terminal of KEIAN M716S V2 with 7 inches display does cost about 7280 Japanese Yen. Major specification and outlook of the Android tablet terminal is shown in Table 3.

TABLE III. SPECIFICATION OF ANDROID TABLET TERMINAL OF KEIAN M716S V2

\begin{tabular}{|c|c|c|}
\hline Dimension & OS & weight \\
\hline $800 \times 480$ & Android 4.4.2 & $305 \mathrm{~g}$ \\
\hline
\end{tabular}

NIR LED is also cheap. Therefore, one set of wild pig monitor does cost about 10000 Japanese Yen. The length of the route in the area in concern is a couple of hundred meters. Therefore, 30 sets of the monitoring system would cover the entire route of wild pigs. The total cost of the hardware system an area is around 300000 Japanese Yen. Obviously it is cheaper than damage cost.

Event driven application software is installed in the Android tablet terminal. When relatively large changes are detected in the current frame compared to the previous frame, the event driven software is activated. Then target object is detected with target extraction and contour extraction. Then size of target object is measured from the contour. Discrimination between adult and cub is done depending on the measured size. After that discrimination between male and female is done depending on presence or absence of nipple.

Along with the suspicious route of wild pigs, the monitoring hardware systems are set every 10 meters. The number of incoming and outgoing wild pigs is counted for each block with 10 meters long. Thus total number of wild pigs can be estimated.

\section{CONCLUSION}

Methods for wild pig identifications and discrimination of female wild pigs based on feature matching methods with acquired Near Infrared: NIR moving pictures are proposed. Trials and errors are repeated for identifying wild pigs and for discrimination of female wild pigs through experiments. As a conclusion, feature matching methods with the target nipple features show a better performance. Feature matching method of FLANN shows the best performance in terms of feature extraction and tracking capabilities.

Further study is required for wide area of spatial distribution of wild pigs. Spatial distribution of wild pigs in a relatively small size of area in concern can be estimated by the proposed system and method. Kiriging can be used for a much wide area in concern using estimated the number of wild pigs of the small size of areas.

\section{ACKNOWLEDGMENT}

The authors would like to thank Mr. Kenji Egashira, Dr. Herman Tolle of Arai's laboratory members for their useful comments and suggestions during this research works.

\section{REFERENCES}

[1] Patten, D. C. 1974. Feral hogs - boon or burden. Proceedings of the Sixth Vertebrate Pest Conference 6:210-234.

[2] Singer, F. J., W. T. Swank, and E. E. C. Clebsch. 1984. Effects of wild pig rooting in a deciduous forest. Journal of Wildlife Management. 48:464-473.

[3] Stone, C. P., and J. O. Keith. 1987. Control of feral ungulates and small mammals in Hawaii's national parks: research and management strategies. Pages 277-287 in C. G. J. Richards and T. Y. Ku, editors. Control of mammal pests. Taylor and Francis, London, England, and New York and Philadelphia, USA.

[4] Cushman, J. H., T. A. Tierney, and J. M. Hinds. 2004. Variable effects of feral pig disturbances on native and exotic plants in a California grassland. Ecological Applications 14:1746-1756.

[5] Kaller, M. D., and W. E. Kelso. 2006. Swine activity alters invertebrate and microbial communities in a coastal plain watershed. American Midland Naturalist 156:163-177.

[6] Bratton, S. P. 1977. The effect of European wild boar on the flora of the Great Smoky Mountains National Park. Pages 47-52 in G. W. Wood, editor. Research and management of wild hog populations. Belle W. Baruch Forest Science Institute, Clemson University, Georgetown, South Carolina, USA.

[7] Lucas, E. G. 1977. Feral hogs - problems and control on National Forest lands. Pages 17-22 in G. W. Wood, editor. Research and management of wild hog populations. Belle Baruch Forest Science Institute, Clemson University, Georgetown, South Carolina, USA.

[8] Thompson, R. L. 1977. Feral hogs on National Wildlife Refuges. Pages 11-15 in G. W. Wood, editor. Research and management of wild hog populations. Belle W. Baruch Forest Science Institute, Clemson University, Georgetown, South Carolina, USA. 
[9] Schley, L, and T. J. Roper. 2003. Diet of wild boar Sus scrofa in Western Europe, with particular reference to consumption of agricultural crops. Mammal Review 33:43-56.

[10] Whitehouse, D. B. 1999. Impacts of feral hogs on corporate timberlands in the southeastern United States. Pages 108-110 in Proceedings of the Feral Swine Symposium, June 2-3, 1999, Ft. Worth, Texas, USA.

[11] Mayer, J. J., E. A. Nelson, and L. D. Wike. 2000. Selective depredation of planted hardwood seedlings by wild pigs in a wetland restoration area. Ecological Engineering, 15(Supplement 1): S79-S85.

[12] Campbell, T. A., and D. B. Long. 2009. Feral swine damage and damage management in forested ecosystems. Forest Ecology and Management 257:2319-2326

[13] Forrester, D. J. 1991. Parasites and diseases of wild mammals in Florida. University of Florida Press, Gainesville, Florida, USA.

[14] Williams, E. S., and I. K. Barker. 2001. Infectious diseases of wild mammals. Iowa State University Press, Ames, Iowa, USA.

[15] Sweeney, J. R., J. M. Sweeney, and S. W. Sweeney. 2003. Feral hog. Pages 1164-1179 in G. A. Feldhamer, B. C. Thompson, and J. A. Chapman, editors. Wild mammals of North America. Johns Hopkins University Press, Baltimore, Maryland, USA.

[16] Nettles, V.F., J. L. Corn, G. A. Erickson, and D. A. Jessup. 1989. A survey of wild swine in the United States for evidence of hog cholera. Journal of Wildlife Diseases 25:61-65.

[17] Davidson, W. R., and V. F. Nettles, editors. 1997. Wild swine. Pages 104-133 in Field manual of wildlife diseases in the southeastern United States. Second edition. Southeastern Cooperative Wildlife Disease Study, Athens, Georgia, USA.

[18] Davidson, W. R., editor. 2006. Wild swine. Pages 105-134 in Field manual of wildlife diseases in the southeastern United States. Third edition. Southeastern Cooperative Wildlife Disease Study, Athens, Georgia, USA.

[19] Choquenot, D. J., R. J. Kilgour, and B. S. Lukins. 1993. An evaluation of feral pig trapping. Wildlife Research, 20:15-22.

[20] Barrett, R. H., and G. H. Birmingham. 1994. Wild pigs. Pages D65-D70 in S. Hyngstrom, R. Timm, and G. Larsen, editors. Prevention and control of wildlife damage. Cooperative Extension Service, University of Nebraska, Lincoln, Nebraska, USA.

[21] Mapston, M. E. 1999. Feral hog control methods. Pages 117-120 in Proceedings of the Feral Swine Symposium, June 2-3, 1999, Fort Worth, Texas, USA.

\section{AUTHORS PROFILE}

Kohei Aarai He received BS, MS and PhD degrees in 1972, 1974 and 1982, respectively. He was with The Institute for Industrial Science and Technology of the University of Tokyo from April 1974 to December 1978 and also was with National Space Development Agency of Japan from January, 1979 to March, 1990. During from 1985 to 1987, he was with Canada Centre for Remote Sensing as a Post Doctoral Fellow of National Science and Engineering Research Council of Canada. He moved to Saga University as a Professor in Department of Information Science on April 1990 He was a councilor for the Aeronautics and Space related to the Technology Committee of the Ministry of Science and Technology during from 1998 to 2000. He was a councilor of Saga University for 2002 and 2003. He also was an executive councilor for the Remote Sensing Society of Japan for 2003 to 2005. He is an Adjunct Professor of University of Arizona, USA since 1998. $\mathrm{He}$ also is Vice Chairman of the Commission-A of ICSU/COSPAR since 2008. He is Editor-in-Chief of IJACSA and IJISA. He wrote 33 books and published 500 journal papers. 